\title{
Long non-coding RNA FOXD2-AS1 promotes proliferation, migration and invasion of ovarian cancer cells via regulating the expression of $\mathrm{miR}-4492$
}

\author{
JIANFEN GAO ${ }^{1}$, FAQIN LIU ${ }^{2}$, XIA ZHAO $^{3}$ and PING ZHANG ${ }^{4}$ \\ ${ }^{1}$ Department of Gynecology; ${ }^{2}$ Operating Room; ${ }^{3}$ Department of Obstetrics, People's Hospital of Zhangqiu District, Jinan, \\ Shandong 250200; ${ }^{4}$ Department of Gynecology, Laizhou People's Hospital, Yantai, Shandong 261400, P.R. China
}

Received April 23, 2019; Accepted September 4, 2019

DOI: $10.3892 /$ etm.2021.9738

\begin{abstract}
The aim of the present study was to determine the role of long non-coding RNA (lncRNA) forkhead box D2 antisense 1 (FOXD2-AS1) in the development of ovarian cancer, investigate the underlying mechanisms and provide a potential diagnostic biomarker for ovarian cancer. A total of 39 ovarian cancer patients were included, and the ovarian cancer tissues and paracancer tissues were obtained. The ovarian cancer cell lines SKOV3 and OVCAR3 and the human ovarian normal epithelial cell line IOSE80 were cultured. The expression of 1ncRNA FOXD2-AS1 and miR-4492 was detected by reverse transcription-quantitative PCR. Small interfering RNA targeting FOXD2-AS1 (si-FOXD2-AS1), microRNA (miR)-4492 mimics, miR-4492 inhibitor and their corresponding controls were transfected into cells. The proliferation was detected with a Cell-Couting-Kit- 8 assay, and migration and invasion were determined using Transwell assays. The mutual binding site of IncRNA FOXD2-AS1 and miR-4492 was predicted with the miRDB database and verified by a luciferase reporter assay. Finally, a rescue assay was performed. The results suggested that lncRNA FOXD2-AS1 was upregulated in ovarian cancer tissues and cell lines. si-FOXD2-AS1 was able to inhibit the proliferation, migration and invasion of ovarian cancer cells. 1ncRNA FOXD2-AS1 was confirmed to directly target miR-4492. The expression of IncRNA FOXD2-AS1 and miR-4492 exhibited a negative correlation. In a rescue experiment, miR-4492 inhibitor abrogated the effect of siFOXD2-AS1 in SKOV3 and OVCAR3 cell lines. In conclusion, IncRNA FOXD2-AS1 promotes the proliferation and invasion of ovarian cancer cells via regulating
\end{abstract}

Correspondence to: Professor Ping Zhang, Department of Gynecology, Laizhou People's Hospital, 1718 Wuli Street, Yantai, Shandong 261400, P.R. China

E-mail:ping_zhanga@163.com

Key words: long non-coding RNA forkhead box D2 antisense 1, microRNA-4492, ovarian cancer, proliferation, migration, invasion the expression of miR-4492. It may be a novel potential diagnostic biomarker and therapeutic target for ovarian cancer.

\section{Introduction}

Ovarian cancer is one of the most common malignant tumor types of the female reproductive system; it has a high incidence rate and poses a serious threat to the lives of females worldwide (1). Ovarian epithelial cancer more commonly occurs in post-menopausal females, while malignant germ cell tumors are more frequently encountered in adolescent or young females (2). The etiology of ovarian malignancies remains elusive and the major reasons are genetic and endocrine factors (3). Ovarian epithelial cancer has no obvious symptoms in the early stage, while $60-70 \%$ of patients with ovarian malignant germ cell tumors are diagnosed and treated in the early stage (4). To date, surgery combined with chemotherapy is the major treatment for ovarian tumors (5). In addition, targeted therapy, endocrine therapy and radiation therapy have certain curative effects (6). However, after treatment, ovarian cancer recurs in numerous patients, and a considerable proportion inevitably die after multiple chemotherapies (7). Therefore, it is important to explore novel therapeutic targets for ovarian cancer treatment.

Long non-coding RNAs (lncRNAs) are a class of RNAs of $200 \mathrm{nt}$ in length, which do not encode proteins (8). They have been indicated to be involved in the regulation of development, differentiation and metabolism, as well as numerous aspects of various diseases (9). To date, a large number of lncRNAs have been confirmed to be involved in the development of ovarian cancer $(10,11)$. For instance, lncRNA human ovarian cancer-specific transcript 2 was reported to regulate the expression of microRNA (miR/miRNA) let-7b and affect the biological behavior of epithelial ovarian cancer cells (10). Furthermore, carboplatin-docetaxel caused upregulation of lncRNA PVT1, which was associated with its anti-ovarian cancer effect (12). Of note, IncRNA FOXD2-AS1 has been intensively researched in recent years and its effect on the development of various cancer types, including small cell lung cancer (13), colorectal cancer (14) and esophageal squamous cell carcinoma (15), has been confirmed. However, the mechanistic roles of IncRNA FOXD2-AS1 in ovarian cancer 
have remained elusive. miR-4492 is also an important factor in cancer. As reported in the study by Boo et al (16), miR-4492 was closely associated with chemoresistance of breast cancer. Furthermore, it also participates in the progression of the anti-viral immune response (17). Therefore, studying the association of lncRNA FOXD2-AS1 and miR-4492 in ovarian cancer may have clinical implications for the diagnosis and treatment of this disease.

In the present study, the expression of lncRNA FOXD2-AS1 and miR-4492 in ovarian cancer tissue and cells was detected and the regulatory interaction was also assessed. More importantly, the effect of 1ncRNA FOXD2-AS1 and miR-4492 in cell proliferation and invasion of ovarian cancer was confirmed. The present study may provide a novel biomarker for the prediction of the disease.

\section{Materials and methods}

Collection of ovarian cancer tissues. A total of 39 ovarian cancer patients (age range, $47 \pm 8.4$ years) undergoing surgery at Laizhou People's Hospital between January 2018 and December 2018 were included. Patients who received radiotherapy or chemotherapy prior to surgery were excluded from the current study. The ovarian cancer tissues and paracancer tissues (at least $3 \mathrm{~cm}$ away from the tumor border and with no microscopic evidence of tumor cells) were obtained and immediately placed in liquid nitrogen for cryopreservation until analysis. All patients provided written informed consent and the study was approved by the ethics committee of Laizhou People's Hospital.

Cell lines and culture. The ovarian cancer cell lines SKOV3, A2780 and OVCAR3, and the human ovarian normal epithelial cell line IOSE80 were purchased from the Shanghai Cell Bank of the Chinese Academy of Sciences. All cells were cultured in RPMI 1640 media with $10 \%$ fetal bovine serum (FBS) and $1 \%$ penicillin/streptomycin, and then cultured at $37^{\circ} \mathrm{C}$ in a humidified incubator with $5 \% \mathrm{CO}_{2}$.

Cell transfection. Ovarian cancer cells (SKOV3 and OVCAR3) in the logarithmic growth phase were inoculated into 6-well cell culture plates. When the confluence of cells reached $30-40 \%$, the transfection was performed according to the instructions of the Lipofectamine 2000 kit (Invitrogen; Thermo Fisher Scientific, Inc.), and small interfering (si)RNA targeting FOXD2-AS1 (si-FOXD2-AS1; 5'-GCGAAGAGU ACGUUGCUAUTT-3'), miR-4492 mimics (5'-GGGGCU GGGCGCGCGCC-3'), miR-4492 inhibitor (5'-GGCGCG CGCCAGCCCC-3') and their corresponding controls (5'-UCA CAACCUCCUAGAAAGAGUAGA-3') were transfected separately. After $24 \mathrm{~h}$ of transfection, the medium was replaced with fresh medium. The transfected siRNAs and miRNAs were synthesized by GenePharma Co. Ltd.

Reverse transcription-quantitative $(R T-q) P C R$ assay. Total RNA was extracted from tissues or cells by using the TRIzol ${ }^{\circledR}$ method (Thermo Fisher Scientific, Inc.). Complementary (c) DNA was obtained by RT using the PrimeScript ${ }^{\text {TM }}$ RT reagent kit (Takara Biotechnology Co., Ltd.). qPCR was performed with the SYBR Green I Supermix (Takara Biotechnology
Co., Ltd.) and quantification was performed using the $2^{-\Delta \Delta \mathrm{Cq}}$ method (18). In brief, $3 \mu \mathrm{g}$ total RNA were reverse-transcribed to cDNA with Moloney murine leukemia virus reverse transcriptase (Invitrogen; Thermo Fisher Scientific, Inc.). The thermocycling conditions were as follows: Denaturation at $95^{\circ} \mathrm{C}$ for $10 \mathrm{~min}$; followed by 40 cycles of denaturation at $95^{\circ} \mathrm{C}$ for $15 \mathrm{sec}$ and elongation at $60^{\circ} \mathrm{C}$ for $1 \mathrm{~min}$. GAPDH and U6 were used as references. The primers were as follows: FOXD2-AS1 forward, 5'-CACTGAGGGACAGCCAAGA-3' and reverse, 5'-GGCGGCGTGTAATTGGTA-3'; GAPDH forward, 5'-GGAGCGAGATCCCTCCAAAAT-3'; miR-4492 forward, 5'-AACGAGACGACGACAGAC-3' and reverse, 5'-GGGGCUGGGCGCGCGCC-3'; U6 forward 5'-CTCGCT TCGGCAGCACA-3' and reverse, 5'-AACGCTTCACGAATT TGCGT-3'.

Cell-Counting-Kit-8 (CCK-8) assay for detecting cell proliferation. si-FOXD2-AS1, miR-4492 and their corresponding controls were transfected into SKOV3 and OVCAR3 cells using Lipofectamine ${ }^{\mathrm{TM}}$ 2000, and cells in the logarithmic growth phase expressing si-FOXD2-AS1 were individually seeded into 96 -well plates $\left(2.0 \times 10^{3}\right.$ cells/well). At 24,48 and $72 \mathrm{~h}, 20 \mu \mathrm{l}$ CCK8 solution was added to each well, followed by incubation for $4 \mathrm{~h}$ at $37^{\circ} \mathrm{C}$. The optical density of each well at $450 \mathrm{~nm}$ was measured with a microplate reader (Berthold Technologies $\mathrm{GmbH} \& \mathrm{Co} \mathrm{KG})$. The optical density values of the four wells were measured, the average value was taken, and the cell growth curve was plotted on the abscissa of the cell culture time. Three parallel wells were set for each sample and the experiment was repeated three times independently.

Migration and invasion assays. Single-cell suspensions of transfected SKOV3 and OVCAR3 cells were prepared and cells were counted. Subsequently, $5 \times 10^{5}$ cells in $100 \mu \mathrm{l}$ RPMI 1640 medium were added to the upper chambers of a Transwell insert. Furthermore, $900 \mu \mathrm{l}$ RPMI-1640 medium containing $20 \%$ fetal bovine serum was added to the lower chamber. For each experimental condition, 3 replicate wells were used. Following culture for $16 \mathrm{~h}$ in a cell incubator, the cells in the upper chamber were wiped off with a cotton swab, and the remaining cells were fixed with $95 \%$ ethanol for $30 \mathrm{~min}$ and stained with $0.5 \%$ crystal violet for $20 \mathrm{~min}$ at room temperature. The chamber was rinsed twice with PBS and dried at room temperature. Images of the migrated cells were captured under the microscope cells were counted. For the invasion experiment, the above protocol was followed with the modification of the $8-\mu \mathrm{m}$ Millicell chamber being coated with Matrigel $^{\circledR}$.

Luciferase reporter assay. The potential binding site between FOXD2-AS1 and miR-4492 was predicted using the miRDB tool (http://mirdb.org/miRDB/index.html). Sequences containing the wild-type (WT) or site-mutated (Mut) region of FOXD2-AS1 were synthesized by Sangon Biotech Co., Ltd., and inserted into the pGL3 vector (Promega Corporation). SKOV3 and OVCAR3 cells were seeded in 24-well plates ( $2 \times 10^{4}$ cells per well) and transfected with the WT or Mut reporter gene vector and $\mathrm{miR}-4492$ mimics or negative controls for $24 \mathrm{~h}$, followed by assessment of the luciferase activity using the dual luciferase assay system (Promega Corp.) according to the manufacturer's protocol. 

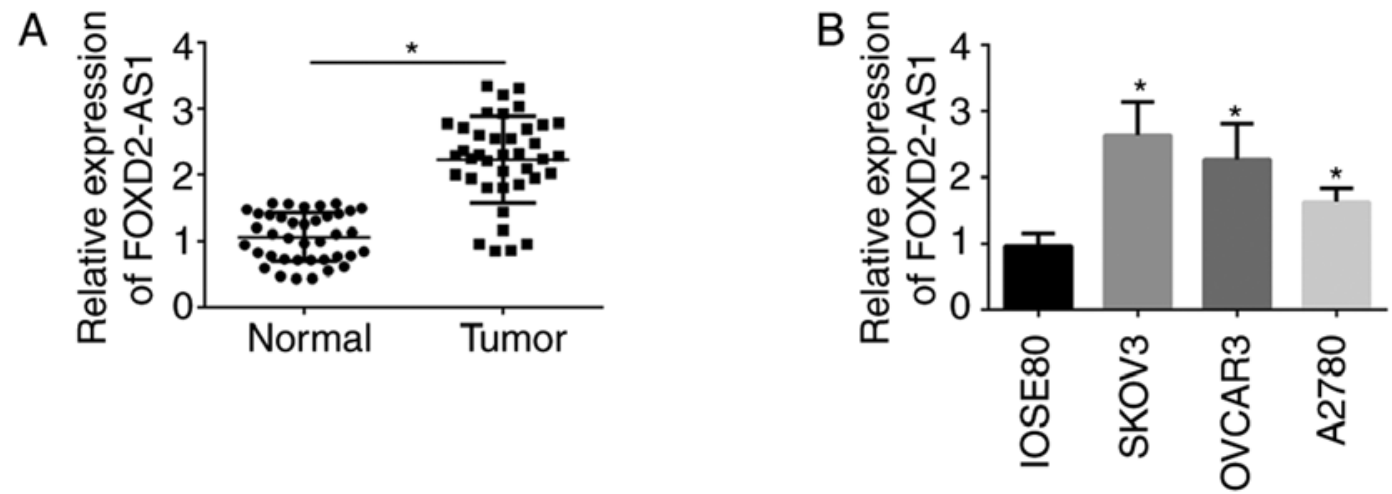

Figure 1. Expression of lncRNA FOXD2-AS1 in ovarian cancer tissues and cell lines. (A) Comparison of lncRNA FOXD2-AS1 in ovarian cancer tissues and paracancer tissues. (B) Levels of lncRNA FOXD2-AS1 in the ovarian cancer cell lines SKOV3, A2780 and OVCAR3, and the human ovarian normal epithelial cell line IOSE80. Reverse transcription-quantitative PCR was used to determine the expression of IncRNA FOXD2-AS1 in ovarian cancer tissues and cell lines. "P<0.05 vs. paracancer tissues/IOSE80. IncRNA, long non-coding RNA; FOXD2-AS1, forkhead box D2 antisense 1.

A
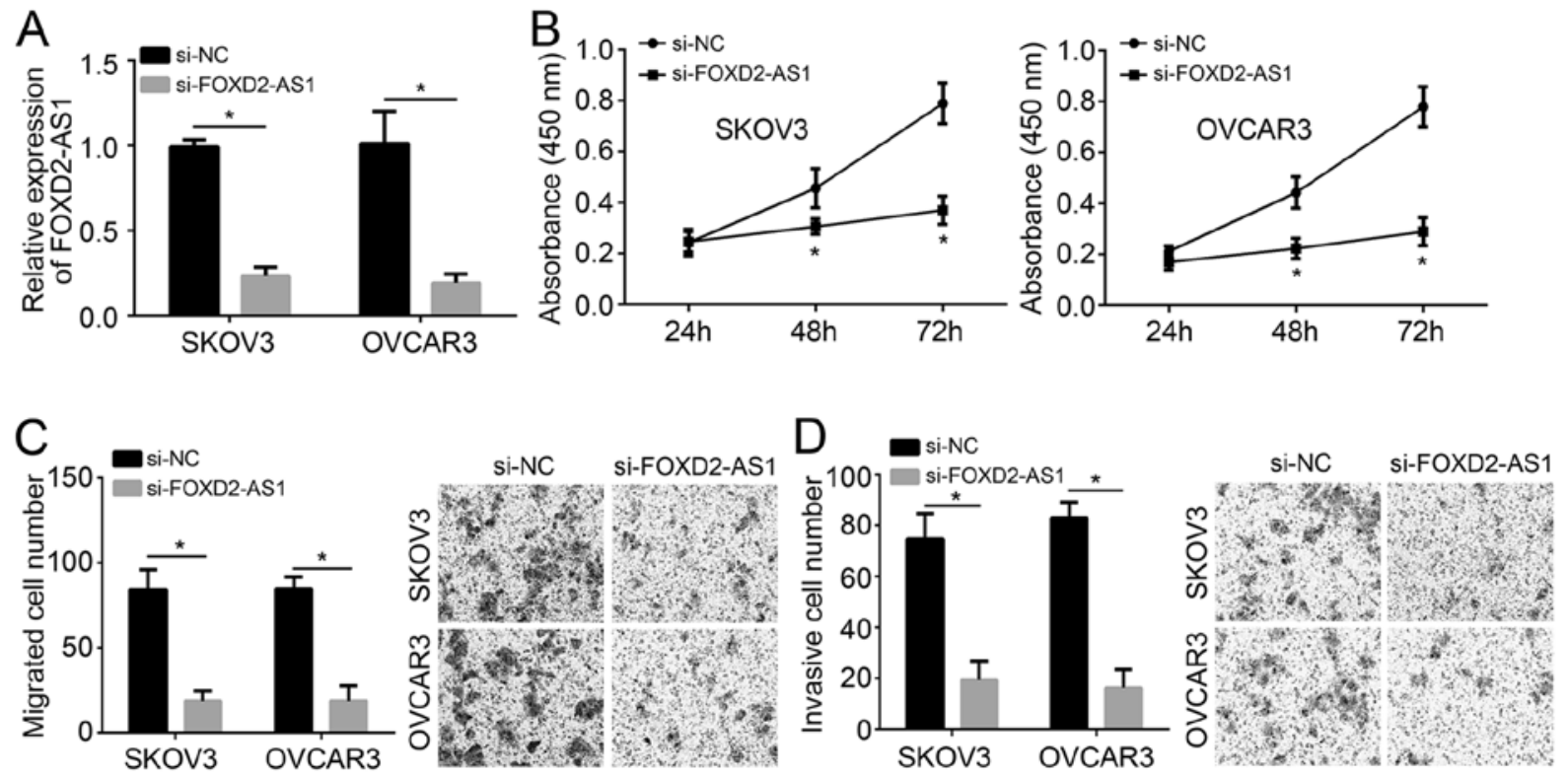

Figure 2. si-FOXD2-AS1 inhibits the proliferation, migration and invasion of ovarian cancer cells. (A) Knockdown efficiency of si-FOXD2-AS1. (B) Proliferation was detected with a CCK-8 assay. (C and D) Transwell assays were performed to assess the (C) migration and (D) invasion of SKOV3 and OVCAR3 cells. (magnification, $\mathrm{x} 100$ ). ${ }^{*} \mathrm{P}<0.05$ vs. si-NC. si-FOXD2-AS1, siRNA targeting forkhead box D2 antisense 1; si-NC, negative control siRNA; siRNA, small interfering RNA; CCK-8, Cell-Counting-Kit-8.

Statistical analysis. Data analysis was performed using SPSS 22.0 software (IBM Corp.). All values are expressed as the mean \pm standard deviation. A Student's t-test was used to analyze the differences between two groups. One-way analysis of variance followed by a Tukey's post-hoc test was used for multiple comparisons. A Pearson's correlation coefficient analysis was used to determine the correlation between expression levels. $\mathrm{P}<0.05$ was considered to indicate a statistically significant difference.

\section{Results}

Expression of IncRNA FOXD2-AS1 in ovarian cancer tissues and cell lines. The expression of 1ncRNA FOXD2-AS1 was detected in a total of 39 paired specimens from ovarian cancer patients. As presented in Fig. 1A, the expression of lncRNA FOXD2-AS1 was significantly higher in ovarian cancer tissues compared with that in paracancer tissues. Similar results were also obtained for the cell lines. Compared with that in the IOSE80 cell line, the expression of IncRNA FOXD2-AS1 was significantly higher in the SKOV3, A2780 and OVCAR3 cell lines (Fig. 1B). In conclusion, lncRNA FOXD2-AS1 was upregulated in ovarian cancer tissues and cell lines.

si-FOXD2-AS1 inhibits the proliferation, migration and invasion of ovarian cancer cells. After transfection with si-FOXD2-AS1, the expression of FOXD2-AS1 was significantly downregulated in SKOV3 and OVCAR3 cell lines (Fig. 2A), which indicated that the knockdown was successful. As indicated by the CCK-8 assay, si-FOXD2-AS1 significantly inhibited the proliferation of ovarian cancer cells after $48 \mathrm{~h}$ $(\mathrm{P}<0.05$; Fig. 2B). In addition, the migration and invasion of si-FOXD2-AS1-transfected SKOV3 and OVCAR3 cells was significantly suppressed (Fig. 2C and D). 

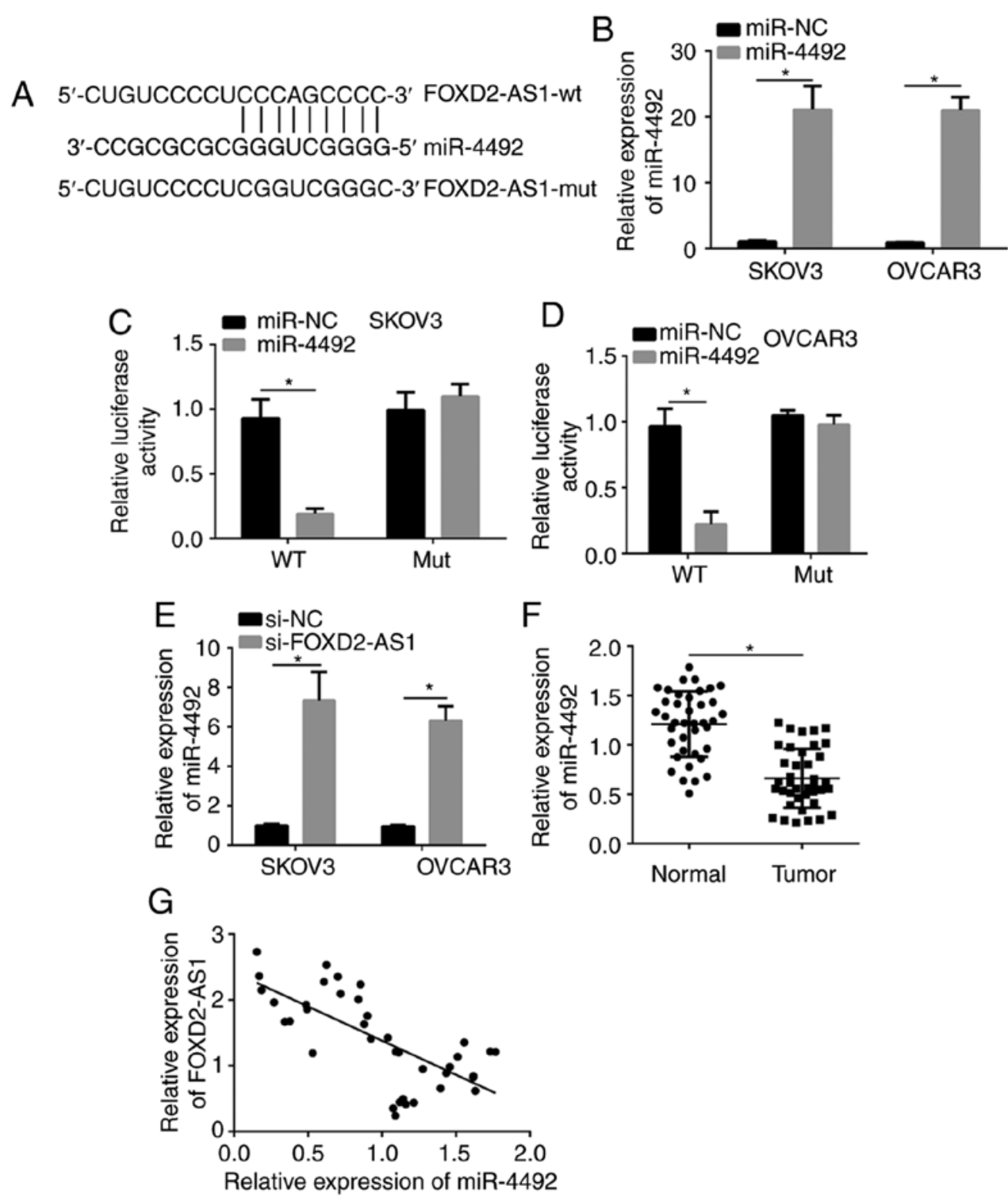

Figure 3. IncRNA FOXD2-AS1 targets miR-4492. (A) The mutual binding site of lncRNA FOXD2-AS1 and miR-4492 was predicted with the miRDB database. (B) RT-qPCR was used to confirm successful transfection of miR-4492 mimics. (C and D) Luciferase reporter assays were performed to confirm the predicted binding site. Relative luciferase activity of lncRNA FOXD2-AS1 in (C) SKOV3 and (D) OVCAR3 cells after miR-4492 transfection. (E) The expression of miR-4492 was significantly upregulated in cells transfected with si-FOXD2-AS1. (F) Relative expression of miR-4492 in pairs of tumor tissues and adjacent normal controls determined by RT-qPCR. (G) The expression of FOXD2-AS1 and miR-4492 was negatively correlated. "P<0.05 as indicated. lncRNA, long non-coding RNA; FOXD2-AS1, forkhead box D2 antisense 1; miR, microRNA; wt, wild-type; mut, mutant; NC, negative control; si-FOXD2-AS1, siRNA targeting FOXD2-AS1; si-NC, negative control siRNA; siRNA, small interfering RNA; RT-qPCR, reverse transcription-quantitative PCR.

LncRNA FOXD2-AS1 targets miR-4492. Previous evidence has demonstrated that lncRNAs act as sponges for miRNAs via interacting with the response elements of their target miRNAs $(19,20)$. Thus,FOXD2-AS1 may be asponge for specific miRNAs. A mutual binding site of lncRNA FOXD2-AS1 and miR-4492 was predicted by using the miRDB database. The predicted complementary sequences are presented in Fig. 3A. To confirm the binding interaction, a luciferase reporter assay was performed. The relative luciferase activity of SKOV3 and OVCAR3 cells transfected with reporter plasmid containing the mutated or wild-type sequence from lncRNA FOXD2-AS1 and co-transfected with miR-4492 mimics or miR-NC was determined. After transfection with miR-4492 mimics, the expression of miR-4492 was significantly increased, indicating that the overexpression was successful (Fig. 3B). When the SKOV3 and OVCAR3 cells were co-transfected with the wild-type reporter plasmid and miR-4492, the luciferase activity was significantly inhibited compared with that in the group co-transfected with miR-NC $(\mathrm{P}<0.05)$, while the luciferase activity of the mutant control vector was comparable in WT and Mut groups ( $\mathrm{P}>0.05$; Fig. $3 \mathrm{C}$ and $\mathrm{D})$. These results demonstrated that lncRNA FOXD2-AS1 reduced the amount of available miR-4492 by binding to the response element of miR-4492, suggesting that lncRNA FOXD2-AS1 directly regulates miR-4492 levels and activity. In addition, the level of miR-4492 was significantly upregulated by si-FOXD2-AS1 (Fig. 3E). Furthermore, miR-4492 was downregulated in tumor vs. paracancerous tissues (Fig. 3F). Of note, the expression of 
A

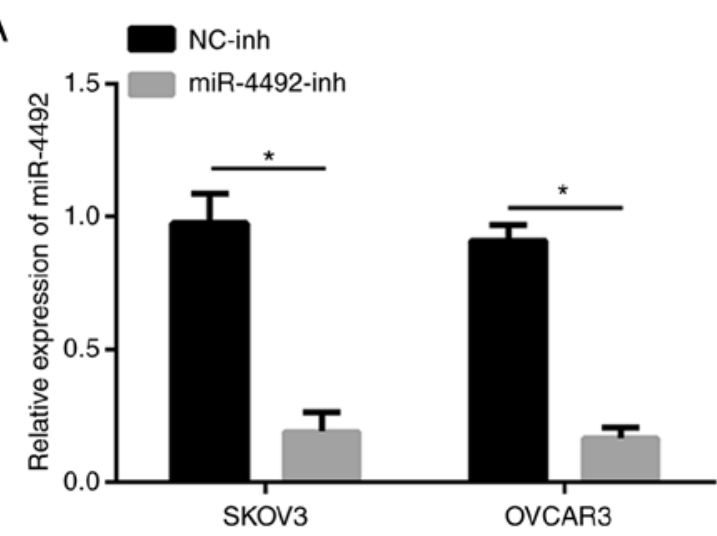

C

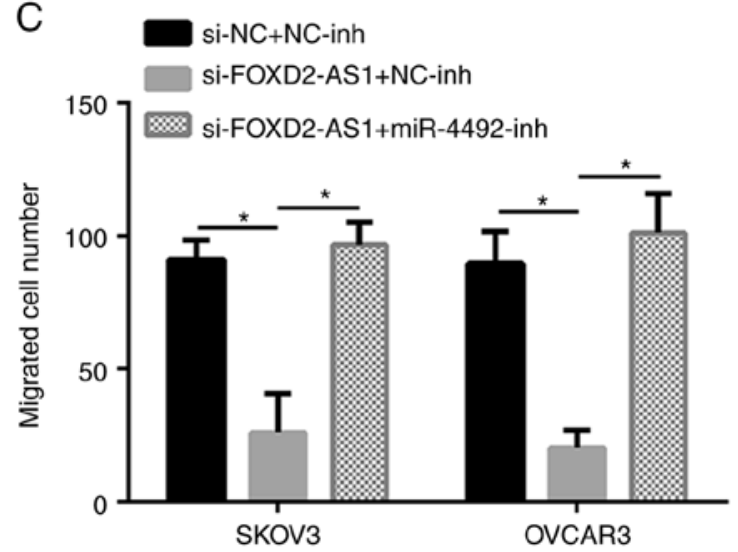

D si-NC+NC-inh

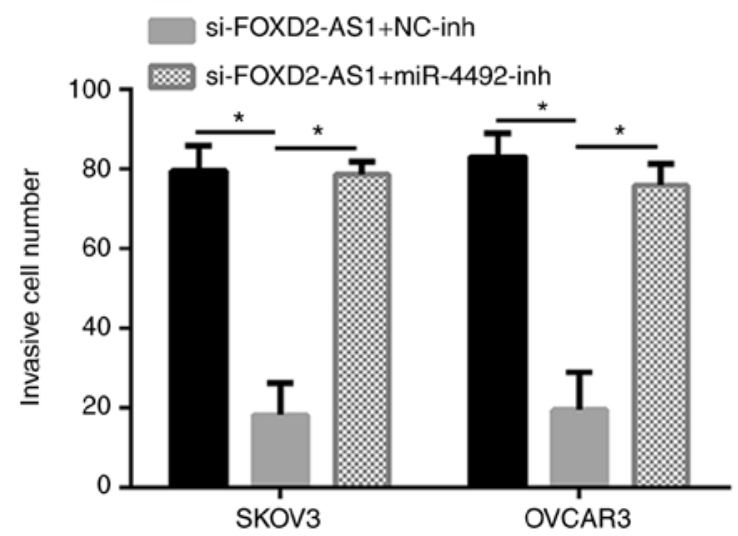

B

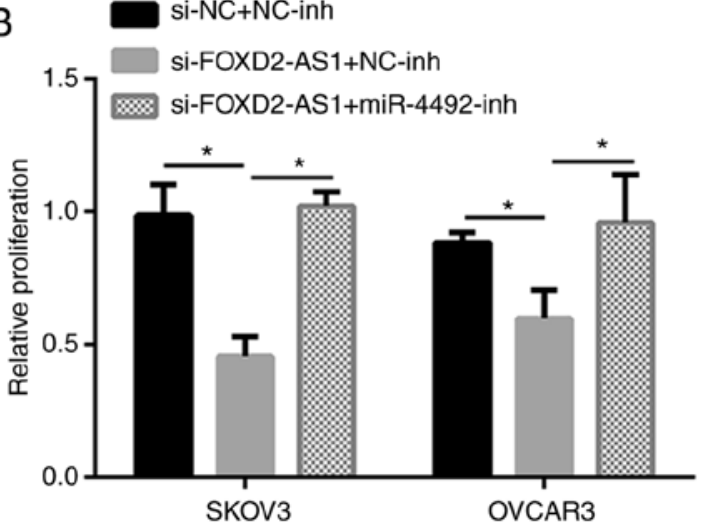

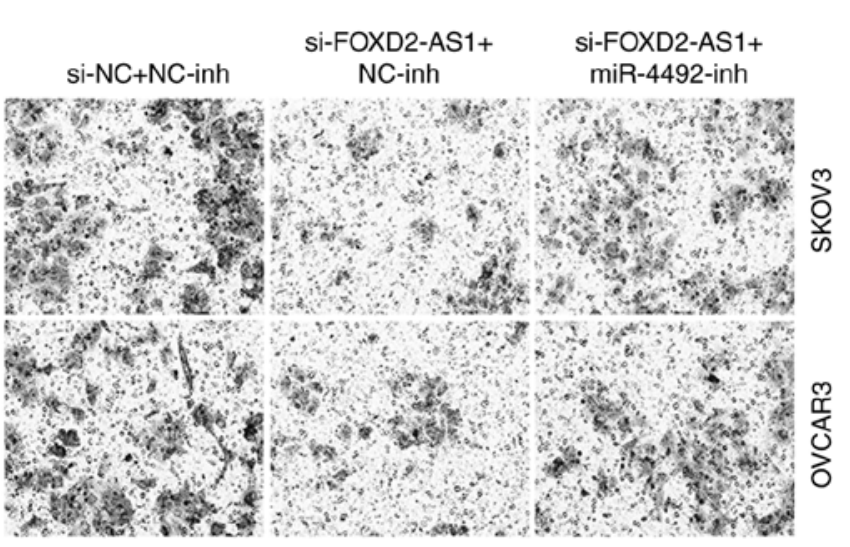

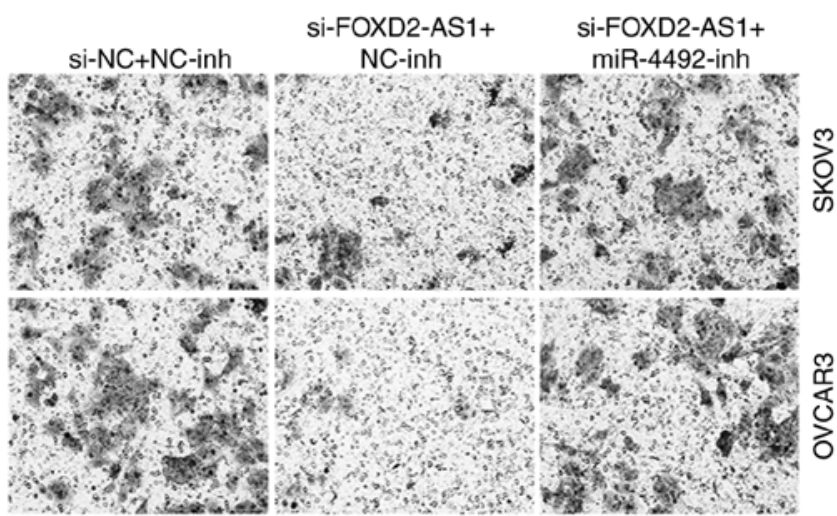

Figure 4. miR-4492 inhibitor rescued the effect of siFOXD2-AS1. (A) Successful inhibition of miR-4492 was confirmed by reverse transcription-quantitative PCR. (B) The proliferation of SKOV3 and OVCAR3 cells was detected with a Cell-Counting-Kit-8 assay. Cells were incubated for $72 \mathrm{~h}$. (C and D) Transwell assays were performed to assess the (C) migration and (D) invasion of SKOV3 and OVCAR3 cells. (magnification, $\mathrm{x} 100$ ). " $\mathrm{P}<0.05$ as indicated. miR, microRNA; NC-inh, negative control of the inhibitor; miR-4492-inh, inhibitor of miR-4492; si-FOXD2-AS1, siRNA targeting forkhead box D2 antisense 1; si-NC, negative control siRNA; siRNA, small interfering RNA.

FOXD2-AS1 and miR-4492 was negatively correlated in tumor tissues ( $r=-0.699$; $\mathrm{P}<0.05$; Fig. 3G).

miR-4492 inhibitor rescues the effect of siFOXD2-AS1. After transfection with miR-4492-inhibitor, the level of miR-4492 was confirmed to be significantly downregulated (Fig. 4A). si-FOXD2-AS1 significantly inhibited the proliferation, migration and invasion of SKOV3 and OVCAR3 cell lines $(\mathrm{P}<0.05)$. However, in a rescue experiment, co-transfection with miR-4492-inhibitor abrogated this effect (Fig. 4B-D). Therefore, miR-4492 inhibitor rescued the effect of
siFOXD2-AS1 to inhibit the proliferation, migration and invasion of SKOV3 and OVCAR3 cell lines.

\section{Discussion}

Ovarian cancer has obvious genetic characteristics and there is a requirement for early diagnostic biomarkers and therapeutic targets (21). Thereby, more accurate biomarkers are urgently required to be screened $(22,23)$. In the present study, the implications of lncRNA FOXD2-AS1 and miR-4492 in the proliferation, migration and invasion of ovarian cancer 
cells were investigated. The results indicated that lncRNA FOXD2-AS1 was highly expressed in ovarian cancer tissues and cells as compared with that in paracancerous tissues and a normal cell line, respectively. siFOXD2-AS1 inhibited the proliferation, invasion and migration of ovarian cancer cells. The expression of lncRNA FOXD2-AS1 and miR-4492 had a negative correlation. Furthermore, miR-4492 inhibitor rescued the suppressive effect of siFOXD2-AS1 on the malignant behavior of ovarian cancer cells.

Previous studies have confirmed that lncRNA FOXD2-AS1 has an important role in various cancer types (13-15). For instance, lncRNA FOXD2-AS1 was confirmed to be highly expressed in non-small cell lung cancer and to participate in the progression of this disease via the Wnt/ $\beta$-catenin signaling pathway (14). Furthermore, it was reported to participate in the Notch signaling pathway and epithelial-to-mesenchymal transition (EMT), and to affect the development of colorectal cancer (15). Of note, the Wnt/ $\beta$-catenin pathway and EMT signaling have been indicated to be important in the development of ovarian cancer $(24,25)$. In addition, An et al (26) confirmed that lncRNA FOXD2-AS1 regulated the expression of miR-143 to thereby improve the gemcitabine resistance of bladder cancer. Of note, miR-143 has been confirmed to inhibit the progression of ovarian cancer by targeting cellular communication network factor 2 (27). Furthermore, lncRNA FOXD2-AS1 was also indicated to regulate the expression of miR-3663-5p and S100A1, and aggravate the genesis of nasopharyngeal carcinoma (28). In ovarian cancer, S100A1 was reported to be highly expressed and to have an important role in cell migration and proliferation (29). For these reasons, lncRNA FOXD2-AS1 was likely to be a key lncRNA in ovarian cancer; however, this has so far remained to be demonstrated. The present study aimed to explore the biological function of lncRNA FOXD2-AS1 in ovarian cancer and the results indicated that the proliferation, as well as the migratory and invasive ability of ovarian cancer cells were decreased after silencing of FOXD2-AS1. This indicates that FOXD2-AS1 may participate in the progression of ovarian cancer, mainly by promoting cell proliferation, migration and invasion.

Of note, IncRNA FOXD2-AS1 was confirmed to have a mutual binding site with miR-4492 in the present study, and miR-4492 inhibitor was able to rescue the suppressive effect of siFOXD2-AS1 in ovarian cancer cells. In 2016, Boo et al (16) published a comprehensive bioinformatics analysis and confirmed that miR-4492 was differentially expressed in breast cancer cells. Furthermore, the expression of miR-4492 was also associated with the immune response $(17,30)$. It has become apparent that the mechanisms of the immune response in ovarian cancer have a significant prognostic significance in the clinic (31). Overall, it is indicated that IncRNA FOXD2-AS1 is able to negatively regulate the levels of miR-4492 and further affect the course of ovarian cancer.

While the implication of 1ncRNA FOXD2-AS1 and miR-4492 in cell proliferation, migration and invasion of ovarian cancer cells was comprehensively assessed in the present study, certain limitations are worth mentioning. First, the regulation at the molecular level is usually complex, and further lncRNAs, genes and miRNAs require to be investigated. In addition, most of the data were obtained from in vitro experiments, and more in vivo experiments, including animal models, require to be performed. Furthermore, the number of clinical samples was small and the results require to be confirmed in more patients. The above issues will be the focus of future research performed by our group. There are also several limitations regarding the molecular biological analysis. For instance, the expression of EMT markers was not measured by western blot analysis, which requires investigation in the future. Furthermore, the downstream targets of miR-4492 were not analyzed, which will also be determined in future work performed by our group.

In conclusion, the present study indicated that IncRNA FOXD2-AS1 promotes the proliferation, migration and invasion of ovarian cancer cells at least partially by regulating the expression of miR-4492. It may potentially serve as a novel diagnostic biomarker and therapeutic target for ovarian cancer.

\section{Acknowledgements}

Not applicable.

\section{Funding}

No funding was received.

\section{Availability of data and materials}

All data generated or analyzed during this study are included in this published article.

\section{Authors' contributions}

JG and PZ contributed to the conception and design of the present study. In addition, PZ analyzed and interpreted the results and wrote the manuscript. FL and XZ performed the experiments. All authors read and approved the final manuscript.

\section{Ethics approval and consent to participate}

The present study was approved by the Ethics Committee of Laizhou People's Hospital. Written informed consent was obtained from all patients enrolled.

\section{Patient consent for publication}

Not applicable.

\section{Competing interests}

The authors declare that they have no competing interests.

\section{References}

1. Golan A, Joosting AC and Orchard ME: Mumps virus and ovarian cancer. S Afr Med J 56: 18-20, 1979.

2. Skates SJ, Menon U, Macdonald N, Rosenthal AN, Oram DH, Knapp RC and Jacobs IJ: Calculation of the risk of ovarian cancer from serial CA-125 values for preclinical detection in postmenopausal Women. J Clin Oncol 21 (10 Suppl): 206s-210s, 2003.

3. Makar AP and Tropé CG: Endometrial and ovarian malignancies: Epidemiology, etiology and prognostic factors. Acta Obstet Gynecol Scand 71: 331-336, 1992. 
4. Du K, Gong HY and Gong ZM: Influence of serum VEGF levels on therapeutic outcome and diagnosis/prognostic value in patients with cervical cancer. Asian Pac J Cancer Prev 15: 8793-8796, 2014.

5. Suzuki M, Ohwara M, Sekiguchi I and Sato I: Radical cytoreductive surgery combined with platinums-carboplatin and cisplatin chemotherapy for advanced ovarian cancer. Int J Gynecol Cancer 9: 54-60, 2010.

6. Slotman BJ and Rao BR: Ovarian cancer (review). Etiology, diagnosis, prognosis, surgery, radiotherapy, chemotherapy and endocrine therapy. Anticancer Res 8: 417-434, 1988.

7. Tu H, Huang H, Huang QD, Li Z, Feng YL and Liu JH: Treatment and prognostic analysis of ovarian cancer patients with isolated region of lymph node recurrence. Zhonghua $\mathrm{Fu}$ Chan $\mathrm{Ke} \mathrm{Za}$ Zhi 47: 928-933, 2012 (In Chinese)

8. Vance KW and Ponting CP: Transcriptional regulatory functions of nuclear long noncoding RNAs. Trends Genet 30: 348-355, 2014

9. Li Y, Zhang J, Huo C, Ding N, Li J, Xiao J, Lin X, Cai B, Zhang Y and $\mathrm{Xu}$ J: Dynamic organization of lncRNA and Circular RNA regulators collectively controlled cardiac differentiation in humans. Ebiomedicine 24: 137-146, 2017.

10. Gao Y, Meng H, Liu S, Hu J, Zhang Y, Jiao T, Liu Y, Ou J, Wang D, Yao L, et al: LncRNA-HOST2 regulates cell biological behaviors in epithelial ovarian cancer through a mechanism involving microRNA let-7b. Hum Mol Genet 24: 841-852, 2015.

11. Chai Y, Liu J, Zhang Z and Liu L: HuR-regulated lncRNA NEAT1 stability in tumorigenesis and progression of ovarian cancer. Cancer Med 5: 1588-1598, 2016.

12. Liu E, Liu Z and Zhou Y: Carboplatin-docetaxel-induced activity against ovarian cancer is dependent on up-regulated lncRNA PVT1. Int J Clin Exp Pathol 8: 3803-3810, 2015.

13. Rong L, Zhao R and Lu J: Highly expressed long non-coding RNA FOXD2-AS1 promotes non-small cell lung cancer progression via Wnt $/ \beta$-catenin signaling. Biochem Biophys Res Commun 484: 586-591, 2017.

14. Yang $X$, Duan B and Zhou X: Long non-coding RNA FOXD2-AS1 functions as a tumor promoter in colorectal cancer by regulating EMT and Notch signaling pathway. Eur Rev Med Pharmacol Sci 21: 3586-3591, 2017.

15. Bao J,Zhou C, Zhang J, Mo J, Ye Q, He J and Diao J: Upregulation of the long noncoding RNA FOXD2-AS1 predicts poor prognosis in esophageal squamous cell carcinoma. Cancer Biomark 21: 527-533, 2018

16. Boo L, Ho WY, Ali NM, Yeap SK, Ky H, Chan KG, Yin WF, Satharasinghe DA, Liew WC, Tan SW, et al: MiRNA transcriptome profiling of spheroid-enriched cells with cancer stem cell properties in human breast MCF-7 cell line. Int J Biol Sci 12: 427-445, 2016.

17. Egaña-Gorroño L, Guardo AC, Bargalló ME, Planet E, Vilaplana E, Escribà T, Pérez I, Gatell JM, García F, Arnedo M, et al: MicroRNA profile in CD8+ T-lymphocytes from HIV-infected individuals: Relationship with antiviral immune response and disease progression. PLoS One 11: e0155245, 2016
18. Livak KJ and Schmittgen TD: Analysis of relative gene expression data using real-time quantitative PCR and the 2(-Delta Delta C(T)) method. Methods 25: 402-408, 2001.

19. Wang A,JinC,LiH, Qin Q and LiL:LncRNA ADAMTS9-AS2regulates ovarian cancer progression by targeting miR-182-5p/FOXF2 signaling pathway. Int J Biol Macromol 120: 1705-1713, 2018.

20. Yan H, Li H, Li P, Li X, Lin J, Zhu L, Silva MA, Wang X, Wang P and Zhang Z: Long noncoding RNA MLK7-AS1 promotes ovarian cancer cells progression by modulating miR-375/YAP1 axis. J Exp Clin Cancer Res 37: 237, 2018.

21. Soegaard M, Kjaer SK, Cox M, Wozniak E, Høgdall E, Høgdall C, Blaakaer J, Jacobs IJ, Gayther SA and Ramus SJ: BRCA1 and BRCA2 mutation prevalence and clinical characteristics of a population-based series of ovarian cancer cases from Denmark. Clin Cancer Res 14: 3761-3767, 2008.

22. Kozak KR, Amneus MW, Pusey SM, Su F, Luong MN, Luong SA, Reddy ST and Farias-Eisner R: Identification of biomarkers for ovarian cancer using strong anion-exchange ProteinChips: Potential use in diagnosis and prognosis. Proc Natl Acad Sci USA 100: 12343-12348, 2003.

23. Tsai MC, Spitale RC and Chang HY: Long intergenic noncoding RNAs: New links in cancer progression. Cancer Res 71: 3-7, 2011.

24. Wang B, Liu M, Zhuang R, Jiang J, Gao J, Wang H, Chen H, Zhang Z, Kuang Y and Li P: Long non-coding RNA CCAT2 promotes epithelial-mesenchymal transition involving Wnt $/ \beta$-catenin pathway in epithelial ovarian carcinoma cells. Oncol Lett 15: 3369-3375, 2018.

25. Usongo M, Li X and Farookhi R: Activation of the canonical WNT signaling pathway promotes ovarian surface epithelial proliferation without inducing $\beta$-catenin/Tcf-mediated reporter expression. Dev Dyn 242: 291-300, 2013.

26. An Q, Zhou L and Xu N: Long noncoding RNA FOXD2-AS1 accelerates the gemcitabine-resistance of bladder cancer by sponging miR-143. Biomed Pharmacother 103: 415-420, 2018.

27. Wang L, He J, Xu H, Xu L and Li N: MiR-143 targets CTGF and exerts tumor-suppressing functions in epithelial ovarian cancer. Am J Transl Res 8: 2716-2726, 2016.

28. Chen G, Sun W, Hua X, Zeng W and Yang L: Long non-coding RNA FOXD2-AS1 aggravates nasopharyngeal carcinoma carcinogenesis by modulating miR-363-5p/S100A1 pathway. Gene 645: 76-84, 2018.

29. Tian T, Li X, Hua Z, Ma J, Liu Z, Chen H and Cui Z: S100A1 promotes cell proliferation and migration and is associated with lymph node metastasis in ovarian cancer. Discov Med 23: 235-245, 2017

30. Xun M, Ma CF, Du QL, Ji YH and Xu JR: Differential expression of miRNAs in enterovirus 71-infected cells. Virol J 12: 56, 2015.

31. Gavalas NG, Karadimou A, Dimopoulos MA and Bamias A Immune response in ovarian cancer: How is the immune system involved in prognosis and therapy: Potential for treatment utilization. Clin Dev Immunol 2010: 791603, 2011. 